\title{
Effects of Post-Exercise Honey Drink Ingestion on Blood Glucose and Subsequent Running Performance in The Heat
}

\author{
Nur Syamsina Ahmad ${ }^{1}$; Foong Kiew Ooi ${ }^{1, *}$; Mohammed Saat Ismail ${ }^{2}$; Mahaneem Mohamed $^{3}$ \\ ${ }^{1}$ Sports Science Unit, School of Medical Sciences, Universiti Sains Malaysia, George Town, Malaysia \\ 2 Exercise and Sports Science Programme, School of Health Sciences, Universiti Sains Malaysia, George Town, Malaysia \\ 3 Department of Physiology, School of Medical Sciences, Universiti Sains Malaysia, George Town, Malaysia \\ ${ }^{*}$ Corresponding author: Foong Kiew Ooi, Sports Science Unit, School of Medical Sciences, University Sains Malaysia, George Town, Malaysia. Tel: +60-97676931, Fax: +60-97641945, \\ E-mail: fkooi@kb.usm.my
}

Received: October 25, 2013; Accepted: March 10, 2014

\begin{abstract}
Background:Glycogen depletion and hypoglycemia have been associated with fatigue and decrement of performance during prolonged exercise

Objectives: This study investigated the effectiveness of Acacia honey drink as a post-exercise recovery aid on glucose metabolism and subsequent running performance in the heat.

Patients and Methods: Ten subjects participated in this randomized cross-over study. All subjects performed 2 trials. In each trial, all subjects went through a glycogen depletion phase (Run-1), 2-hour rehydration phase and time trial running phase (Run-2). In Run-1, subjects were required to run on a treadmill at $65 \% \mathrm{VO}_{2 \mathrm{max}}$ in the heat $\left(31^{\circ} \mathrm{C}, 70 \%\right.$ relative humidity) for 60 min. During 2-hour rehydration phase, subjects drank either plain water (PW) or honey drink (HD) with amount equivalent to $150 \%$ of body weight loss in 3 boluses (60\%, $50 \%$ and $40 \%$ subsequently) at 0,30 and $60 \mathrm{~min}$. In Run-2, the longest distance covered in 20 min was recorded for determining running performance. Two-way repeated measured ANOVA and paired t-test were used for analysis.

Results: Running distance in Run-2 covered by the subjects in the honey drink HD trial $(3420 \pm 350 \mathrm{~m})$ was significantly $(\mathrm{P}<0.01)$ longer compared to plain water PW trial $(3120 \pm 340 \mathrm{~m})$. In general, plasma glucose, serum insulin and osmolality were significantly $(\mathrm{P}<0.05)$ higher in HD compared to PW during the rehydration phase and Run-2.

Conclusions: These findings indicate that rehydration with honey drink improves running performance and glucose metabolism compared to plain water in the heat. Thus, honey drink can be recommended for rehydration purpose for athletes who compete in the heat.
\end{abstract}

Keywords: Ingestion; Exercise; Blood Glucose

\section{Background}

Glycogen depletion and hypoglycemia have been associated with fatigue and decrement of performance during prolonged exercise (1). Ingestion of fluid containing carbohydrate $(\mathrm{CHO})$ can improve exercise performance by preventing dehydration $(2,3)$ and reducing the decline in blood glucose (4). Carbohydrate can be ingested in a bolus feeding or dispersed in intervals. It has been mentioned that intake of $\mathrm{CHO}$ in the first 2 hours after exercise allows fast rate of glycogen synthesis (5), and fluid should be consumed directly proportional or close to sweat loss to maintain important physiological functions (2). Additionally, if water is consumed, the volume ingested needs to exceed the fluid deficit by approximately $150 \%$ to compensate for the urinary loss that occurs with water ingestion $(6,7)$. Honey is one of the carbohydrate sources. To date, the possible role of carbohydrate contained in honey during recovery i.e. after exercise is still unclear. Thus, the present study was proposed. Acacia honey used in the present study was obtained from the Johor region, Malaysia, and it was produced by Apis mellifera bees. Based on the laboratory analysis done in department of Molecular Medicine, Universiti Malaysia, Malaysia, it was reported that Acacia honey contains $31.2 \%$ of fructose, $22.9 \%$ of glucose, $3.3 \%$ of maltose and $9.9 \%$ of sucrose, it also contains $13 \mathrm{mg}$ of sodium per $100 \mathrm{~g}$ of serving and $75 \mathrm{~g}$ of $\mathrm{CHO}$ per $100 \mathrm{~g}$ of serving. It was speculated that honey consists of different types of carbohydrate, and these multiple transportable carbohydrates may can lead to high carbohydrate oxidation rates and result in better performance during exercise. This speculation was based on a recent previous study which showed that a mixture of glucose and fructose ingestion resulted in approximately 55\% higher exogenous carbohydrate oxidation rates compared to the ingestion of an isocaloric amount of glucose during prolonged cycling exercise (8). Additionally, in another previous study, it was found that a mixture of glucose, sucrose and fructose ingestion resulted in higher ex-

Copyright ( 2015, Sports Medicine Research Center. This is an open-access article distributed under the terms of the Creative Commons Attribution-NonCommercial 4.0 International License (http://creativecommons.org/licenses/by-nc/4.0/) which permits copy and redistribute the material just in noncommercial usages, provided the original work is properly cited. 
ogenous carbohydrate oxidation rates compared with glucose ingestion alone during 150 min cycling (9). The beneficial effects of glucose and fructose supplementation on endurance performance was also shown in a meta analysis done by Vandenbogaerde and Hopkins (2011) (10), in which it was found that the best supplement inferred from the analysis consisted of a $\sim 3-10 \%$ carbohydrate-plus-protein drink providing $\sim 0.7 \mathrm{~g} / \mathrm{kg} / \mathrm{h}$ glucose polymers, $\sim 0.2 \mathrm{~g} / \mathrm{kg} / \mathrm{h}$ fructose and $\sim 0.2 \mathrm{~g} / \mathrm{kg} / \mathrm{h}$ protein. According to Ivy (1998) (4), fructose contained in honey is beneficial for the replenishment of liver glycogen. Consumption of fructose or sucrose during recovery may increase the supply of glycogen substrate to the liver and thus increase the relative proportion of whole body glycogen resynthesis occuring within the liver (11). Moreover, ingestion of CHO drink can maintain a higher osmolality of blood effectively compared to plain water (12). In a recent review paper by Memon and Kumar (2013) (13), it was mentioned that fructose ingestion may produce deleterious effects on the cardiovascular system, such as increase in blood pressure and also elicit adverse metabolic effects, for example insulin resistance and hypertriglyceridaemia. However, fructose which is contained in natural sources such as honey may produce beneficial effects on human health. In addition, it was mentioned in White's study (2013) (14) that fructose intake at normal population levels but not hyperdosing does not cause biochemical outcomes substantially different from other dietary sugars. To date, the sports drinks available in the market are mostly carbonated drinks which may cause gastric discomfort. Acacia honey drink which will be prescribed to the subjects in the present study is not a carbonated drink. It is believed that this non carbonated honey drink may not cause gastric discomfort, while it is ingested by subjects after exercise.

\section{Objectives}

Since the efficacy of Acacia honey drink, a non carbonated drink, as a post-exercise recovery aid on glucose metabolism and subsequent running performance in the heat has not been attempted before, therefore the present study was proposed. If the present study can confirm that Acacia honey drink could elicit beneficial effects on glucose metabolism and subsequent running performance in the subjects, it then can be proposed to the athletes for enhancing their sport performance in a hot and humid environment.

\section{Patients and Methods}

Ten male recreational runners (age: $21.8 \pm 1.4$ years old; body weight: $59.9 \pm 7.8 \mathrm{~kg} ; \mathrm{VO}_{2 \max }: 51.7 \pm 4.1 \mathrm{~mL} . \mathrm{kg}-1 . \mathrm{min}$ 1) who were able to run on the treadmill at $65 \% \mathrm{VO}_{2 \max }$ for at least 60 min were recruited as subjects in this study. They were healthy with no chronic diseases or taking any medication. Subjects were asked to refrain from ingesting any products containing honey for 48 hours before the experiment trials. Subjects were also asked to refrain from strenuous exercise for 24 hours prior to the actual experimental trials. A food diary form was given to each subject to record their food intake three days before the experimental trial. They were required to repeat the same diet over three days before the consecutive experimental trial to minimise differences in muscle glycogen. Subjects were also asked to fast from 10.00 p.m. onwards on the day before the experimental trial. However, they were permitted to drink plain water. The study was approved by the Research and Ethics Committee of School of Medical Sciences, University Sains Malaysia (USM), Kelantan, Malaysia (Reference number: USMKK/PPP/JEPeM [228.3.(040)]).

\subsection{Preliminary Tests}

After subjects' recruitment, the subjects were required to perform two preliminary tests, i.e. (i) A 16-min incremental submaximal running test to determine the relationship between running speed and oxygen uptake, followed by (ii) $\mathrm{A} \mathrm{VO}_{2 \max }$ test, which was an uphill incremental treadmill running to exhaustion test for determining each subject's $\mathrm{VO}_{2 \max }$. From the data obtained in the submaximal running test and $\mathrm{VO}_{2 \max }$ test, the speed which elicited $50 \% \mathrm{VO}_{2 \max }$ and $65 \% \mathrm{VO}_{2 \max }$ of the subject were calculated, so that the appropriate running intensity used in the actual experimental trials could then be determined. In order to familiarise subjects with the experimental protocol, subjects performed a familiarisation trial one week before the actual experimental trial, in which they were required to run in the heat $\left(31^{\circ} \mathrm{C}, 70 \%\right.$ relative humidity) at $65 \% \mathrm{VO}_{2 \max }$ on a motorised treadmill for 60 minutes.

\subsection{Experimental Trial, Honey Drink and Environ- mental Condition}

On the actual experimental trial days, subjects reported to the laboratory at $8 \mathrm{a} . \mathrm{m}$. after a 10-hour overnight fast. The following procedures were carried out accordingly before the commencement of each trial: (i) A standardised breakfast with two pieces of white bread and $500 \mathrm{ml}$ of plain water one hour before each trial; (ii) Determination of nude body weight by using an electronic body composition analyser (Tanita ( TBF-410, Japan); and (iii) Cannulation for blood sample drawing. All subjects performed 2 trials. In each trial, subjects went through three phases, i.e. 60 min of glycogen depletion phase (Run-1), followed by 2-hour rehydration phase and time trial running phase (Run-2). Immediately before warm up of Run-1, blood samples were collected. Subsequently, subjects were asked to warm-up for $5 \mathrm{~min}$ by running at $50 \% \mathrm{VO}_{2 \max }$. Immediately after the completion of the warm-up, the intensity of running was increased to $65 \%$ $\mathrm{VO}_{2 \max }$. During Run-1, blood samples were collected at 0 -min and at the end of the 60 min running. After com- 
pleting Run-1, subjects' post-exercise nude body weights were obtained after they had towel-dried themselves for determining the body weight loss. Subsequently, the subjects were required to rest for 2 hours during the rehydration phase. During this period, the subjects drank either plain water (PW) or honey drink (HD) with an amount equivalent to $150 \%$ of body weight loss in 3 boluses ( $60 \%, 50 \%$ and $40 \%$ of body weight loss respectively) at 0,30 and $60 \mathrm{~min}$. Both the drinks which were kept at $8^{\circ} \mathrm{C}$ were prepared by the laboratory technologist. These drinks were given to the subjects in a random order. The blood samples were collected at every $30 \mathrm{~min}$ for 2 hours during rehydration phase. Fluid sensation such as thirst, sweetness, nausea, fullness and stomach upset were determined using a fluid sensation scale (15) each time after fluid ingestion. Then, subjects were required to run again at $50 \% \mathrm{VO}_{2 \max }$ for 5 min and immediately followed by a 20 min-time trial (Run-2). In Run-2, the longest distance covered in 20 min by the subjects was recorded as the endurance running performance. Blood samples were taken before and after warm up, and at the end of the time trial. Subjects came to the lab after 7 days from the first trial for their second trial. For the second trial, the experimental protocol was similar to the first trial. The present study was a single blind study, thus the researcher did not know about the type of drink the subjects were prescribed during each experimental trial. This randomization and distribution was done by a laboratory technologist. In the present study, the honey drink contained $6.8 \%$ of $\mathrm{CHO}$ which was equivalent to the $\mathrm{CHO}$ concentration in most sports drinks available in the market. During the experimental trials, the laboratory was maintained at a hot environmental temperature of $31^{\circ} \mathrm{C}$ and relative humidity of $70 \%$.

\subsection{Blood Analysis}

Four ml of venous blood was drawn from the subjects during each blood collection. One $\mathrm{ml}$ of the blood was transferred into a tube anticoagulated with natrium fluoride (NaF). The blood in the tube was separated by centrifugation $\left(10 \mathrm{~min}, 400 \mathrm{rpm}, 4^{\circ} \mathrm{C}\right.$ ). The plasma obtained was stored at $-20^{\circ} \mathrm{C}$ for subsequent analysis of glucose concentration using a commercially-available reagent kit (Randox, United Kingdom) and a chemistry analyser (Selectra E, The Netherlands). Two ml of blood was transferred into a plain tube and was then separated by centrifugation. Serum was stored at $-20^{\circ} \mathrm{C}$ for analysis of serum osmolality and insulin. Serum osmolality was analysed using cryoscopic osmometer (Osmomat 030, Gonotec, Germany) while serum insulin was analysed using electrochemiluminescence immunoassay 'ECLIA' kits (Roche, US). For the analysis of haematocrit levels, the blood sample was placed in the Ethylenediamine tetra-acetic acid (EDTA) tubes. Blood haematocrit levels were determined via a microhematocrit centrifuge (Hettich-Haematocrit 20, German) and a reader (Hawksley, England).

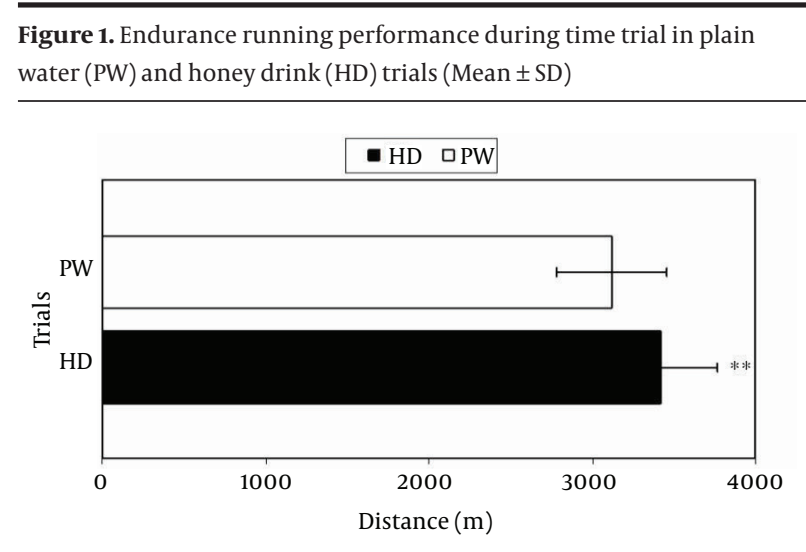

**, $\mathrm{P}<0.01$ compared to PW trial; The running distance of HD trial was significantly $(\mathrm{P}<0.01)$ longer than the $\mathrm{PW}$ trial.

\subsection{Statistical Analysis}

Statistical analysis was performed using statistical software contained in the Statistical Package for Social Sciences (SPSS) version 18.0. Two-way repeated measure ANOVA with Post hoc Bonferroni test was used to determine the differences of the measured parameters between trials at each time point and the changes of the measured parameters over the time. In the present study, changes of body weight, total volume of fluid consumed, fluid sensation, and blood haematocrit level were analysed by using two way ANOVA with repeated measures. Meanwhile, time trial running performance was analysed using paired-t test. Statistical significance was accepted at $\mathrm{P}<0.05$. Results are expressed as means \pm standard deviation (SD).

\section{Results}

\subsection{Running Performance}

Graphical illustration of the endurance running performance is presented in Figure 1. The present study showed that the running distance covered by the subjects in HD trial $(3420 \pm 350 \mathrm{~m})$ was significantly $(\mathrm{P}<0.01)$ longer than the PW trial $(3120 \pm 340 \mathrm{~m})$.

\subsection{Environmental Condition}

Mean room temperature and relative humidity were similar between PW and HD trials. Room temperature in the PW and HD trials was $30.9 \pm 0.3$ and $31.1 \pm 0.3^{\circ} \mathrm{C}$ respectively while relative humidity was 70.2 \pm 0.4 and $70.4 \pm 0.3$ $\%$ respectively. Changes of body weight, total volume of fluid consumed, fluid sensation, and blood haematocrit level: Subjects' mean pre body weight for PW and HD trials was $59.21 \pm 7.9 \mathrm{~kg}$ and $59.44 \pm 8.0 \mathrm{~kg}$ respectively. The mean post body weight of the subjects after Run-1 was $58.31 \pm 7.7 \mathrm{~kg}$ in PW trial and $58.57 \pm 7.7 \mathrm{~kg}$ in HD trial. The mean percent body weight loss as a result of $60 \mathrm{~min}$ dehydration exercise was $1.50 \pm 0.5 \%$ and $1.54 \pm 0.5 \%$ of the pre body weight in the PW and HD trials respectively. 
Table 1. Fluid Sensation Scale for Thirst, Sweetness, Nausea, Fullness and Stomach Upset in Plain Water (PW) and Honey Drink (HD) Trials (Mean \pm SD) $)^{\mathrm{a}, \mathrm{b}, \mathrm{c}}$

\begin{tabular}{|c|c|c|c|}
\hline \multirow[t]{2}{*}{ Drink } & \multicolumn{3}{|c|}{ Time (Minutes) of the 2-Hour Rehydration Phase } \\
\hline & $\mathbf{0}$ & 30 & 60 \\
\hline \multicolumn{4}{|c|}{ Thirst ( $1=$ not thirsty; $5=$ extremely thirsty) } \\
\hline PW & $2.4 \pm 1.35$ & $1.6 \pm 0.84$ & $1.6 \pm 1.27$ \\
\hline HD & $2.8 \pm 1.14$ & $2.3 \pm 1.34$ & $1.7 \pm 0.95$ \\
\hline \multicolumn{4}{|c|}{ Sweetness $(1=$ not sweet; 5 = extremely sweet) } \\
\hline PW & $1.2 \pm 0.63$ & $1.2 \pm 0.63$ & $1.2 \pm 0.63$ \\
\hline HD & $3.1 \pm 0.74^{\mathrm{C}}$ & $3.2 \pm 0.63^{\mathrm{C}}$ & $3.2 \pm 0.63^{C}$ \\
\hline \multicolumn{4}{|c|}{ Nausea $(1=$ no nausea; 5 = extremely nausea $)$} \\
\hline PW & $1.9 \pm 0.99$ & $2.1 \pm 1.10$ & $2.1 \pm 1.20$ \\
\hline HD & $1.4 \pm 0.52$ & $1.6 \pm 0.52$ & $1.6 \pm 0.52$ \\
\hline \multicolumn{4}{|c|}{ Fullness ( 1 = no fullness; 5 = extremely fullness $)$} \\
\hline PW & $2.7 \pm 1.06$ & $3.1 \pm 0.88$ & $3.6 \pm 0.97$ \\
\hline HD & $1.8 \pm 0.79$ & $2.6 \pm 1.17$ & $3.3 \pm 1.34$ \\
\hline \multicolumn{4}{|c|}{ Stomach upset $(1=$ not upset; 5 = extremely upset $)$} \\
\hline PW & $2.0 \pm 0.94$ & $2.0 \pm 1.25$ & $2.2 \pm 1.23$ \\
\hline HD & $1.7 \pm 0.82$ & $1.9 \pm 0.99$ & $1.9 \pm 0.74$ \\
\hline
\end{tabular}

${ }^{\mathrm{a}}$ Abbreviations: PW, plain water; HD, Honey Drink.

${ }^{\mathrm{b}}$ Sensation of sweetness was significantly higher $(\mathrm{P}<0.001)$ in HD than PW during the recovery phase.

${ }^{\mathrm{C}}$ significantly different from corresponding value in $\mathrm{PW}$ trial at $\mathrm{P}<0.001$.

The rehydrated body weight achieved after 2 hour of rehydration period was $58.96 \pm 8.0 \mathrm{~kg}$ and $59.28 \pm 8.1 \mathrm{~kg}$ for PW and HD trials respectively. There were no significant differences between the two trials in all the above mentioned parameters. The total volume of fluid consumed by the subjects during rehydration period was $1350 \pm 524.4 \mathrm{~mL}$ and $1395 \pm 574.7 \mathrm{~mL}$ in PW and HD trial respectively, with no statistically significant differences between them. Subject's responses to fluid sensation are shown in Table 1. There were no significant differences between PW and HD trials for thirst, nausea, fullness and stomach upset throughout the 2-hour recovery phase. However, at 0-min, 30-min and 60-min of the recovery phase, there were significant differences $(P<0.001)$ for sensation of sweetness between HD and PW, where HD showed higher values than PW trial. Resting value of the blood haematocrit level of PW and HD trials was $43.8 \pm$ $1.7 \%$ and $44.3 \pm 1.7 \%$ respectively, and there was no significant difference in the resting value of the blood haematocrit level between the two trials. In both the PW and HD trials, blood haematocrit levels significantly $(P<0.01)$ increased over time in Run-1 with values of PW: $45.6 \pm 1.7$ $\%$ and HD: $46.0 \pm 1.4 \%$ at the end of Run-1, and the levels of this measured parameter decreased significantly $(\mathrm{P}<$ 0.05 ) over time in the rehydration phase. Haematocrit level at the end of rehydration phase for PW and HD trials was $43.0 \pm 2.7 \%$ and $43.7 \pm 1.4 \%$ respectively. Subsequently, haematocrit levels increased continuously until the end of Run-2 (PW: $46.2 \pm 2.1 \%$; HD: $46.9 \pm 1.5 \%$ ) in both the trials. There were no significant differences in haematocrit levels at any time point between the two trials.

\subsection{Plasma Glucose, Serum Insulin and Serum Os- molality}

Results of plasma glucose are presented in Figure 2. In the glycogen depletion phase (Run-1), no significant difference was observed in plasma glucose between HD and PW trials. Nevertheless, for each trial, there was a non statistically significant decrease in plasma glucose level from beginning until the end of Run-1. During rehydration phase, plasma glucose in HD trial increased significantly at the first 30 min compared to resting value ( $0 \mathrm{~min}$ ) and reduced to a normal level at the end of rehydration phase (120 min). In PW trial, plasma glucose level was lower than resting value throughout the rehydration phase. During Run-2, trends of increase in plasma glucose concentrations until the end of the run were observed in both HD and PW trials. Serum insulin concentrations are shown in Figure 3. There were non statistically significant decreases in serum insulin concentrations in both HD and PW trials during Run-1. During the rehydration phase, no significant changes were observed in serum insulin in PW trial. However, in HD trial, the levels of serum insulin at 30, 60, 90 and 120 min were significantly higher than the value at the end of Run-1 and also significantly higher than PW trial at corresponding times. Reduced serum insulin level was also observed in HD trial at the later stage of rehydration phase. At the end of time trial running phase (Run-2), serum insulin concentration was significantly decreased in HD trial. However, there was no significant difference in serum insulin concentrations at the end of Run-2 compared to respective end of rehydration phase in PW trial. Results of serum osmolality are 
illustrated in Figure 4. In HD trial, serum osmolality level at 30, 60, 90 and 120 min during rehydration phase were significantly lower than the value at the end of Run-1. In PW trials, serum osmolality levels at 60, 90 and 120 min during rehydration phase were significantly lower than the value at the end of Run-1. At 60 min of rehydration phase, serum osmolality level was significantly higher $(\mathrm{P}<0.05)$ in HD than PW. At the end of Run-2, serum osmolality levels in both HD and PW trial were significantly higher than the value at the end of rehydration phase respectively.

Figure 2. Plasma Glucose Concentration (mmol.L-1) During Run-1, Rehydration and Run-2 of Plain Water (PW) and Honey Drink (HD) Trials (Mean \pm SD)

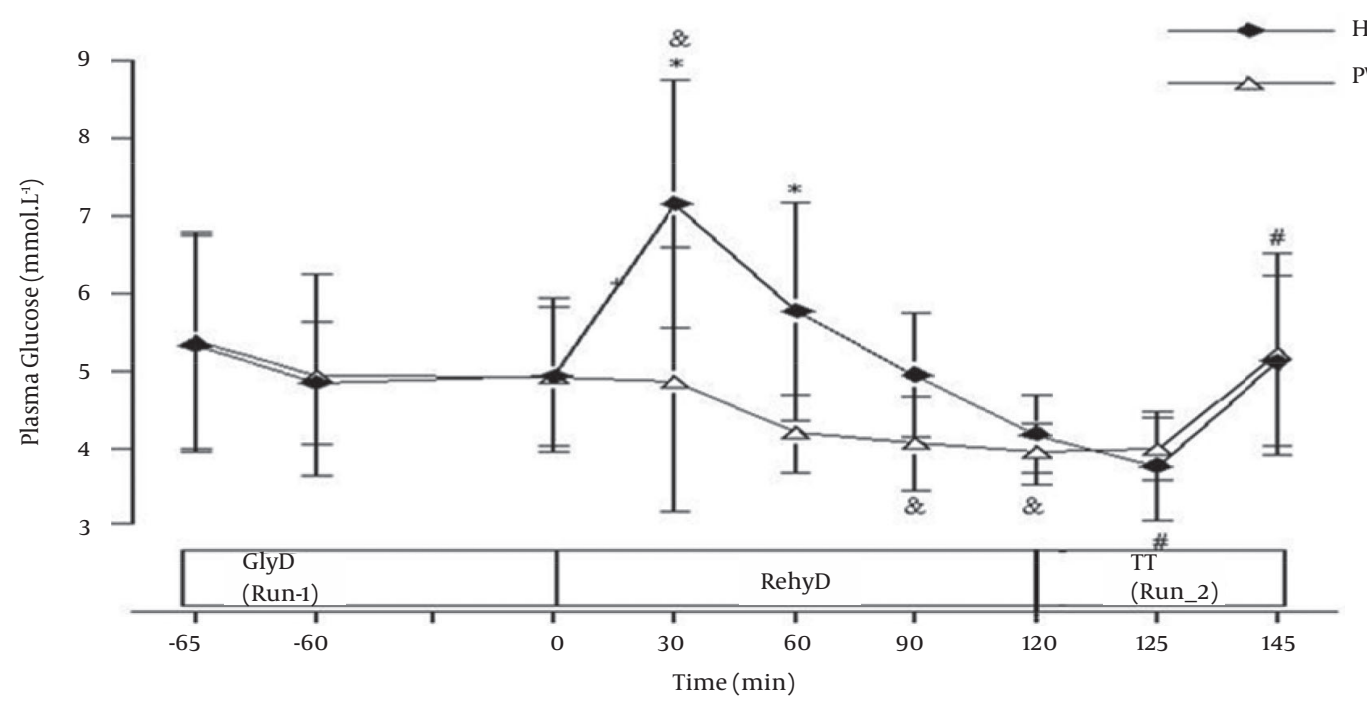

GlyD, Glycogen depletion phase; RehyD, Rehydration phase; TT, Time trial phase; ${ }^{\&} \mathrm{P}<0.05$ compared to respective end of Run-1; ${ }^{\#}$ P $<0.05$ compared to respective end of rehydration phase; ${ }^{*} \mathrm{P}<0.05$ compared to corresponding time in $\mathrm{PW}$ trial; Plasma glucose was significantly higher $(\mathrm{P}<0.05)$ than respective end of Run-1 in HD at 30 min of rehydration phase; Plasma glucose concentrations in PW were significantly lower $(\mathrm{P}<0.05)$ than respective end of Run-1 at 90 minutes and end of rehydration phase.

Figure 3. Serum Insulin Concentration (pmol.L-1) During Run-1, Rehydration and Run-2 of Plain Water (PW) and Honey Drink (HD) Trials (Mean \pm SD)

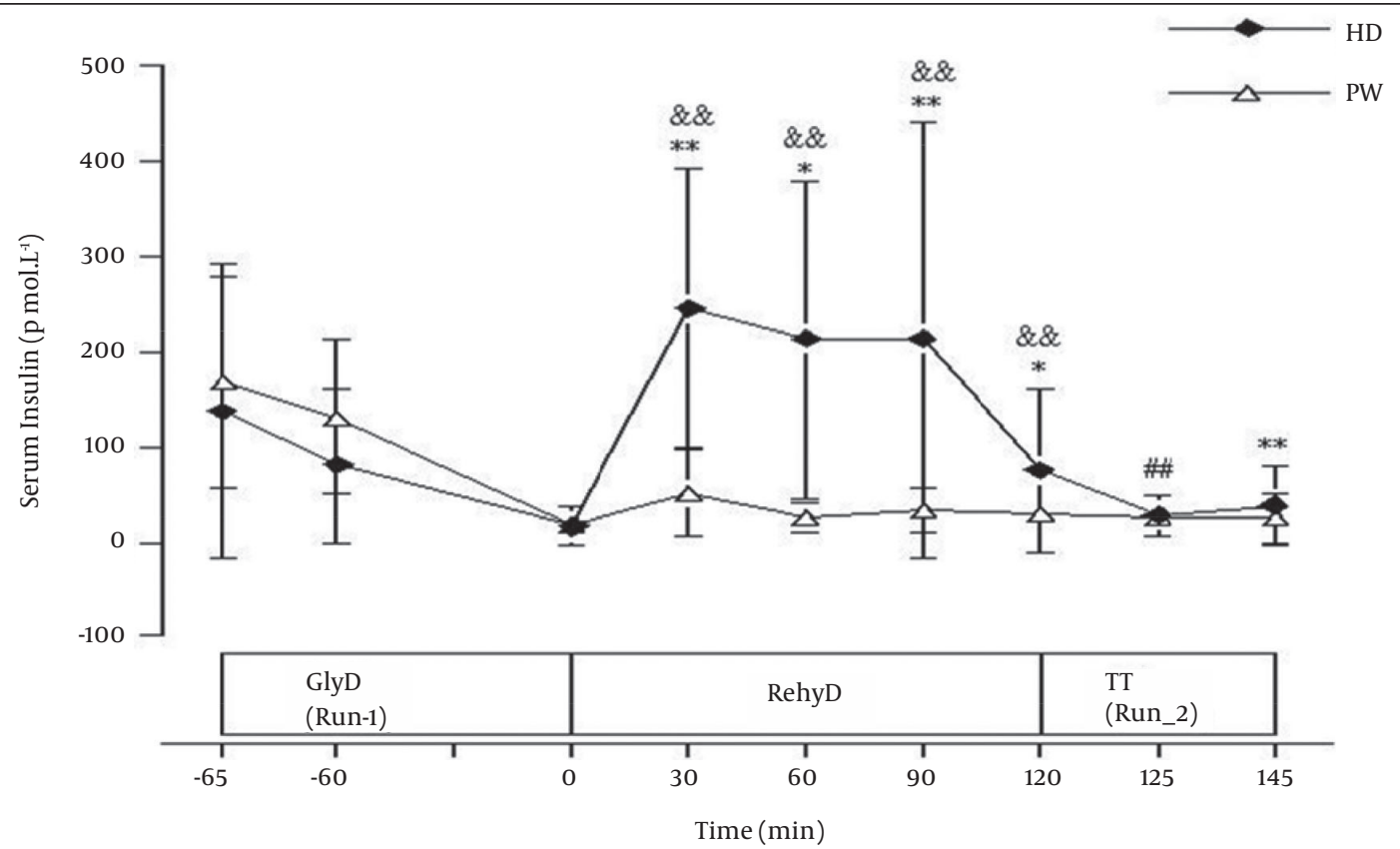

GlyD, Glycogen depletion phase; RehyD, Rehydration phase; TT, Time trial phase; ${ }^{\&} \& \mathrm{P}<0.01$ compared to respective end of Run-1; ${ }^{\# \#} \mathrm{P}<0.01$ compared to respective end of rehydration phase; ${ }^{* *} \mathrm{P}<0.05$ and $\mathrm{P}<0.01$ compared to corresponding time in PW trial; In HD, serum insulin levels at $30,60,90$ and 120 min of rehydration phase were significantly higher $(\mathrm{P}<0.01)$ than the value at the end of Run-1, and also significantly higher $(\mathrm{P}<0.05)$ than $\mathrm{PW}$ trial at corresponding times. 


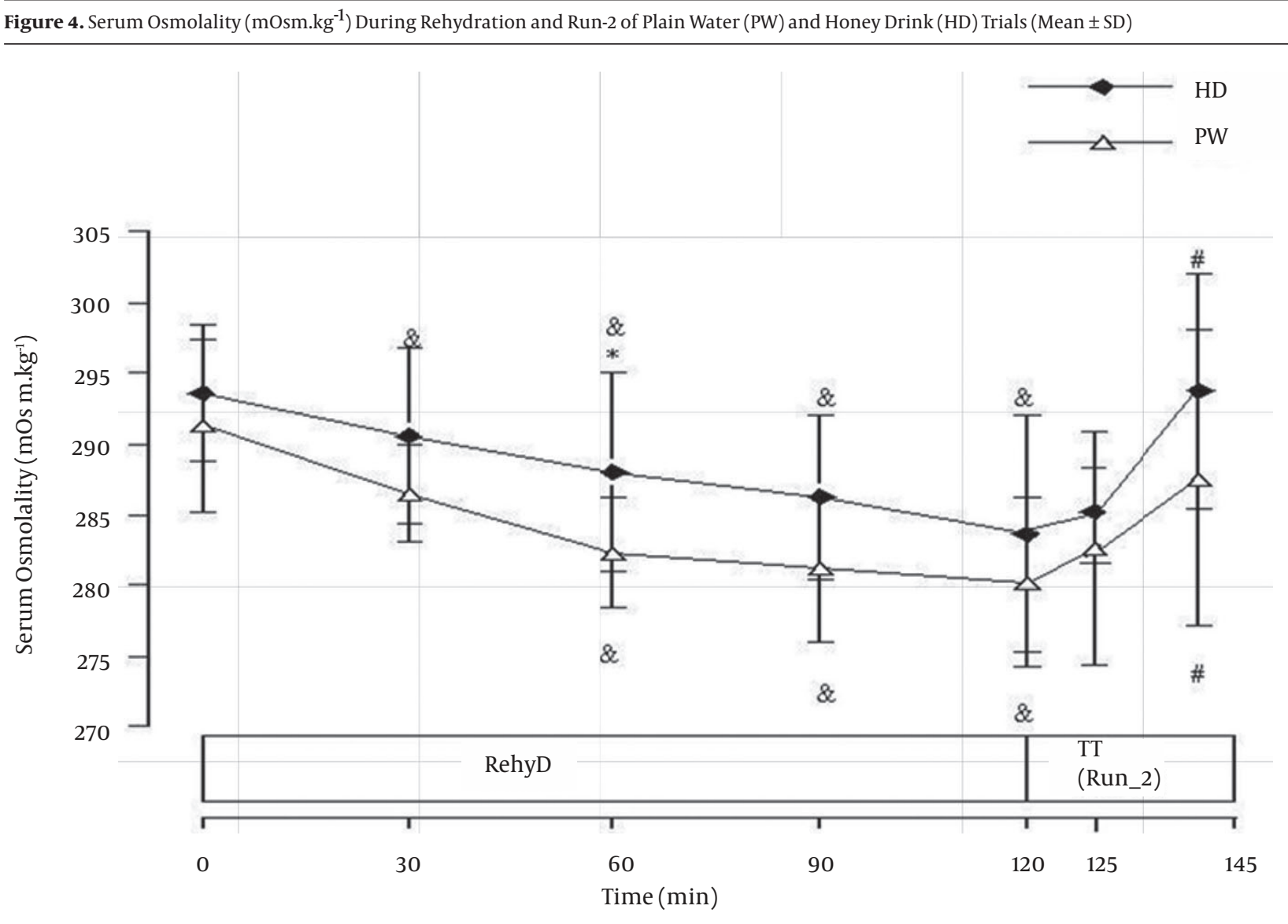

GlyD, Glycogen depletion phase; RehyD, Rehydration phase; TT, Time trial phase; ${ }^{\&} \mathrm{P}<0.05$ compared to respective end of Run-1; ${ }^{\#} \mathrm{P}<0.05$ compared to respective end of rehydration phase; $\mathrm{P}<0.05$ compared to corresponding time in PW trial; At 60 min of rehydration phase, serum osmolality level was significantly higher $(\mathrm{P}<0.05)$ in HD than PW.

\section{Discussion}

The notable finding of the present study is that rehydration with Acacia honey drink could improve running performance in the heat compared to plain water. Additionally, ingestion of Acacia honey drink could elicit significantly higher values of plasma glucose, plasma insulin and serum osmolality compared to plain water during the rehydration phase and time trial running performance. It is generally known that loss of body weight caused by sweating can elicit negative effects on exercise outcome, impairing thermoregulation and performance of prolonged aerobic exercise. These effects are most pronounced when exercise is undertaken in a hot environment. Sawka et al. (16) reported that sport performance can be adversely affected even with $1 \%$ body weight loss in $60 \mathrm{~min}$ of intense exercise at $31^{\circ} \mathrm{C}$. In the present study, the average percent body weight loss after 60 min of dehydration exercise was $1.50 \pm 0.5 \%$ and $1.54 \pm$ $0.5 \%$ in the PW and HD trials respectively. After ingesting $150 \%$ of body weight loss during the rehydration phase, there were no significant differences in post rehydration body weight in the subjects between the trials. Similarly, there were no significant differences in total volume of fluid consumed between the trials. These results demonstrated that the subjects were equally well rehydrated with either PW or HD. In the present study, it was found that there were no significant differences between PW and HD trials for thirst, nausea, fullness and stomach upset throughout the 2-hour rehydration phase. Cool and sweetened beverages are believed to be able to enhance the palatability of the fluid. However, HD elicited significantly $(\mathrm{P}<0.05)$ higher rate of sweetness than PW trial at $0 \mathrm{~min}, 30 \mathrm{~min}$ and $60 \mathrm{~min}$ of the recovery phase. These results suggested that the $8^{\circ} \mathrm{C}$ cooled and sweet Acacia honey drink did not cause a sensation of nausea, fullness and most importantly it did not cause stomach upset in the subjects. The mean blood haematocrit values were not significantly different at the beginning of both PW and HD trials. This suggests that the subjects' preexisting level of hydration were similar at the beginning of each trial. Blood haematocrit levels increased significantly during Run-1 and Run-2, and decreased significantly during rehydration phase in both the trials. These results 
indicated that subjects in Run-1 and Run-2 had reduced plasma volume, and subjects were rehydrated with increased plasma volume during rehydration phase in both PW and HD trials. The absence of any significant differences in blood haematocrit levels at any time point between the two trials implied that the hydration status of the subjects was similar in both the PW and HD trials. Thus, the endurance running performance of both trials was not affected by the hydration status of subjects. It is speculated that the $\mathrm{CHO}$ present in Acacia honey may have played its role for enhancing running performance in the present study. The improved running performance of the subjects in the HD trial could be attributed to the maintenance of blood glucose level during running caused by the presence of $\mathrm{CHO}$ contained in Acacia honey drink consumed by the subjects during the rehydration phase. In a previous study done by Earnest et al. (17) which investigated the effects of honey gel consumption during long distance cycling in a thermoneutral environment, it was observed that honey gel ingestion during cycling could enhance sports performance. The positive finding of both Earnest et al. (17) and the present study suggested that honey may serve as an ergogenic aid for athletes who train and complete either in thermoneutral or hot environments. According to Bowtell et al. (11), the efficiency of $\mathrm{CHO}$ in promoting resynthesis of the body carbohydrate stores is dependent on the insulin and glucose responses to CHO load. This is related to the functions of gastric emptying and intestinal absorption rates, as well as the insulinogenic potential of the $\mathrm{CHO}$, as indicated by the glycemic index of $\mathrm{CHO}$ (18). Acacia honey has been reported to have a low glycemic index (19). In this present study, subjects ran for $60 \mathrm{~min}$ at $65 \%$ $\mathrm{VO}_{2 \mathrm{max}}$ before honey ingestion, and it was observed that there were trends of reduction in plasma glucose level in both honey and plain water trials (Figure 2), reflecting reduction of glucose concentrations following exercise in the subjects. However, when the subjects ingested Acacia honey drink during the rehydration phase, plasma glucose level increased significantly in the first 30 min of rehydration phase, while increase of plasma glucose level was not observed with plain water ingestion during rehydration phase. This observation implies that Acacia honey drink, but not plain water could increase plasma glucose especially during the initial stage of rehydration phase. According to Jentjens et al. (20), rapid muscle glycogen synthesis is characterised by an exercise-induced translocation of glucose transporter carrier protein- 4 to the cell surface, leading to an increased permeability of muscle membrane to glucose. Therefore, it is speculated that Run-1 may have caused an increased rate of glucose transport and an increased capacity to convert glucose into glycogen, and resulting in reduction of plasma glucose concentration at the later stage of rehydration phase in the present study. It is also speculated that the greater elevated level of plasma glucose concentration following the ingestion of low glycemic index Acacia honey drink compared to plain water during the rehydration phase could have contributed to the better running performance with honey drink ingestion in the present study. The carbohydrates contained in Acacia honey consumed in the present study are believed to be appropriate for maintaining a high level of blood glucose and subsequent high insulin level during rehydration phase (Figure 3) which in turn ensures better running performance during subsequent time trial in HD trial. A previous study done by Judelson et al. (21) also found a significantly higher level of serum insulin concentration during rehydration phase to match the rise of glucose level in the blood. Higher level of glucose potently stimulates glycogenesis activity for the homeostasis process (22). It was mentioned by Kochan et al. (23) that muscle recovery from glycogen depletion may be influenced by the availability of insulin. In the present study, insulin level increased and then decreased gradually towards the end of rehydration phase in HD trial. The increased serum insulin level may have caused transport of $\mathrm{CHO}$ to the muscle for replenishing muscle glycogen at the early stage of the rehydration phase in the subjects. Reduction of insulin level at the later stage of rehydration phase in response to low level of plasma glucose may have caused adequate glucose store as an energy booster in the subjects. In the present study, it is suggested that intestinal uptake of carbohydrate contained in Acacia honey drink to the blood stream of the subjects might explain the observation of higher serum osmolality in HD trial compared to PW trial without carbohydrate ingestion (Figure 4), and the $\mathrm{CHO}$ present in Acacia honey may have contributed to the improved running performance in HD trial. The limitations of the present study were that urine analysis was not carried out to confirm the rehydration status of the subjects, and another drink containing a similar concentration of carbohydrate as honey should be used as test drink for comparison purposes. Rehydration with honey drink elicited greater beneficial effects compared to plain water in enhancing blood glucose, insulin and osmolality, as well as endurance running performance in the heat. Thus, Acacia honey drink can be recommended as an ergogenic aid for rehydration purposes in athletes who train and compete in the heat. Nevertheless, further study with urine analysis for confirming the rehydration status of the subjects is warranted. In addition, comparison between honey drink with another drink containing carbohydrate in their effects on sports performance is proposed to be carried out as our future study.

\section{Acknowledgements}

Thanks to Assoc. Professor Siti Amrah for arranging this sponsorship. We would also like to express our sincerest gratitude to Mrs. Jamaayah Binti Meor Osman, Mrs. Nur Hafizah Hamzah, Mrs. Norlida binti Azalan and Mrs Parimalah Velo for their assistance in this study. 


\section{Financial Support}

This study was financially supported by a short term research grant (304/PPSP/61310058) provided by Universiti Sains Malaysia. The Acacia honey used in the present study was sponsored by An-Nur Benut Pontian Company, Malaysia.

\section{References}

1. Tsintzas K, Williams C. Human muscle glycogen metabolism during exercise. Effect of carbohydrate supplementation. Sports Med.1998;25(1):7-23.

2. Murray R. Rehydration strategies--balancing substrate, fluid, and electrolyte provision. Int J Sports Med. 1998;19 Suppl 2:S133-5.

3. Jeukendrup AE. Nutrition for endurance sports: marathon, triathlon, and road cycling.J Sports Sci. 2011;29 Suppl 1:S91-9.

4. Ivy JL. Glycogen resynthesis after exercise: effect of carbohydrate intake. Int J Sports Med. 1998;19 Suppl 2:S142-5.

5. Coyle EF. Timing and method of increased carbohydrate intake to cope with heavy training, competition and recovery. J Sports Sci. 1991;9 Spec No:29-51.

6. Sharp RL. Role of sodium in fluid homeostasis with exercise. JAm Coll Nutr. 2006;25(3 Suppl):231S-9S.

7. Shirreffs SM, Maughan RJ. Rehydration and recovery of fluid balance after exercise. Exerc Sport Sci Rev. 2000;28(1):27-32.

8. Jentjens RL, Jeukendrup AE. High rates of exogenous carbohydrate oxidation from a mixture of glucose and fructose ingested during prolonged cycling exercise. Br J Nutr. 2005;93(4):485-92.

9. Jentjens RL, Achten J, Jeukendrup AE. High oxidation rates from combined carbohydrates ingested during exercise. Med Sci Sports Exerc. 2004;36(9):1551-8.

10. Vandenbogaerde TJ, Hopkins WG. Effects of acute carbohydrate supplementation on endurance performance: a meta-analysis. Sports Med. 2011;41(9):773-92.

11. Bowtell JL, Gelly K, Jackman ML, Patel A, Simeoni M, Rennie MJ. Effect of different carbohydrate drinks on whole body carbo- hydrate storage after exhaustive exercise. J Appl Physiol (1985). 2000;88(5):1529-36.

12. Cheuvront SN, Carter R, Montain SJ, Stephenson LA, Sawka MN Influence of hydration and airflow on thermoregulatory control in the heat. J Therm Biol. 2004;29(7-8):471-7.

13. Memon MQ, Kumar A. The fructose mystery: how bad or good is it? PakJ Pharm Sci. 2013;26(6):1241-5.

14. White JS. Challenging the fructose hypothesis: new perspectives on fructose consumption and metabolism. Adv Nutr. 2013;4(2):246-56

15. Kiew OF, Singh R, Sirisinghe RG, Suen AB, Jamalullail SM. Effects of a herbal drink on cycling endurance performance. Malays $J$ Med Sci. 2003;10(1):78-85.

16. Sawka MN, Pandolf KB. Effects of body water loss on physiological function and exercise performance. In: GCLD editor. Perspectives in exercise science and sports medicine.. Indianapolis: Benchmark Press; 1990.

17. Earnest CP, Lancaster SL, Rasmussen CJ, Kerksick CM, Lucia A, Greenwood MC, et al. Low vs. high glycemic index carbohydrate gel ingestion during simulated $64-\mathrm{km}$ cycling time trial performance. J Strength Cond Res. 2004;18(3):466-72.

18. Burke LM, Collier GR, Hargreaves M. Muscle glycogen storage after prolonged exercise: effect of the glycemic index of carbohydrate feedings. JAppl Physiol (1985). 1993;75(2):1019-23.

19. Ischayek JI, Kern M. US honeys varying in glucose and fructose content elicit similar glycemic indexes. J Am Diet Assoc. 2006;106(8):1260-2.

20. Jentjens R, Jeukendrup A. Determinants of post-exercise glycogen synthesis during short-term recovery. Sports Med. 2003;33(2):117-44.

21. Judelson DA, Maresh CM, Yamamoto LM, Farrell MJ, Armstrong LE, Kraemer WJ, et al. Effect of hydration state on resistance exercise-induced endocrine markers of anabolism, catabolism, and metabolism. J Appl Physiol (1985). 2008;105(3):816-24.

22. Gropper SS, Smith JL. Advanced nutrition and human metabolism. 5th edUSA: Wadsworth; 2008

23. Kochan RG, Lamb DR, Lutz SA, Perrill CV, Reimann EM, Schlender KK. Glycogen synthase activation in human skeletal muscle: effects of diet and exercise. Am J Physiol. 1979;236(6):E660-6. 